\title{
Marcadores genéticos en hipertensión esencial
}

\author{
CRISTÓBAL PASSALACQUA ${ }^{1}$, SILVIA CASTILLO TAUCHER ${ }^{1,2}$
}

\section{Genetic markers in essential hypertension}

Essential hypertension (HTA) is a multifactorial disease and in Chile, its prevalence is $33.7 \%$. There is a genetic predisposition to develop hypertension, whose magnitude is approximately 30 to 50\%. At present, some factors are known to increase the risk for cardiovascular disease, but widely accepted biomarkers for screening are missing. The first studies that looked for candidate genes have focused on the reninangiotensin - aldosterone, aducina, adrenoreceptors $\beta, G$ protein subunits, G protein signaling regulators, kinases associated with $G$ proteins and Rho kinases. Studies of DNA sequencing, search for polymorphisms and variants through single nucleotide polymorphisms, have been used to seek partnerships with complex or multifactorial diseases, like HTA. Examples of these are: components of collagen proteins, genes related to cell myocardial proteins belonging to cytochrome $P 450$ and growth factors, among others. It is still unlikely to count in a near future with a universal marker. Most probably, a series of markers that confer susceptibility to a specific individual will have to be used in prevention programs or personalized therapy.

(Rev Med Chile 2010; 138: 767-772).

Key words: Gene components; Heredity; Hypertension.

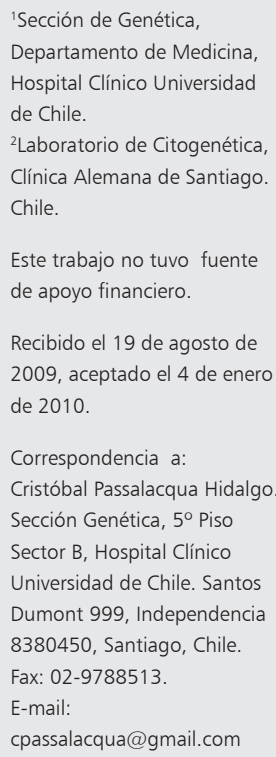

L a hipertensión esencial (HTA), es una enfermedad multifactorial, ampliamente difundida por el mundo, representa el factor de riesgo más importante para desarrollar daño cardiovascular, como: enfermedad cerebrovascular, enfermedad coronaria, enfermedad vascular periférica y falla renal progresiva.

En Chile, la prevalencia general de HTA según encuesta del MINSAL $2005^{1}$, es de $33,7 \%$, siendo la prevalencia en el grupo etario mayor de 65 años de $78,8 \%$, con una tasa de mortalidad por enfermedad hipertensiva en Chile de 16,9/100.000 habitantes el 2003.

Debido a la trascendencia del problema se han intentado diversas aproximaciones, con el fin de lograr una mayor comprensión de la enfermedad. Dada la rápida expansión que ha tenido la genética en los últimos años, ésta se ha transformado en una importante herramienta, aplicable no sólo a enfermedades de herencia mendeliana sino también a enfermedades multifactoriales.
En Chile, trabajos pioneros como Cruz-Coke et $\mathrm{al}^{2}$ relacionaron cómo los componentes genéticos y su interacción con factores ambientales modifican la presión arterial.

Los biomarcadores son sustancias que pueden ser medidas en los niveles molecular, bioquímico o celular, que, al ser interpretadas, se relacionan con una determinada situación biológica o patológica.

Desde una perspectiva clínica, los biomarcadores tienen una variedad de funciones, diferentes a cada etapa en el desarrollo de una enfermedad. Pueden ayudar en el cuidado de pacientes sin enfermedad aparente (biomarcadores de tamizaje), en aquellos con sospecha de tener la enfermedad (biomarcadores diagnósticos) y en aquellos con la enfermedad manifiesta (biomarcadores pronósticos).

En la actualidad, hay biomarcadores cardiovasculares disponibles, diagnósticos y pronósticos, pero faltan biomarcadores de tamizaje ampliamente aceptados, que podrían tener un 
Tabla 1. Glosario

Citocromo: grupo de enzimas que participan en el metabolismo de los medicamentos, que se ubican en mayores concentraciones en el hígado

ELISA: sigla correspondiente a enzimo-inmuno-análisis, utilizado para la detección de enfermedades, sustancias, etc., a través de un marcador fluorescente

Frecuencia: relación de personas afectadas por una característica con la población general

Monogénico: producido por un solo gen, se analoga a mendeliano

Prevalencia: porcentaje de la población que está afectada de una enfermedad en un momento determinado

Polimorfismo: existencia de más de un alelo normal, donde el menos frecuente es superior al $1 \%$ de la población.

Polimorfismos de nucleótido simple (SNPs): es una variación en la secuencia de ADN, que afecta a una sola base del genoma

Repeticiones cortas en tándem (STR): cuando una secuencia de dos o más nucleótidos repetidos están adyacentes entre sí

Variante: cambios en la secuencia de ADN diferentes a las existentes que se mantiene en el tiempo

impacto substancial en la carga de salud pública por la posibilidad de la prevención. Las tecnologías emergentes han sido integradas en los esfuerzos de búsqueda de biomarcadores clínicamente útiles.

Las limitaciones de los biomarcadores disponibles están dadas por no considerar la importancia de identificar biomarcadores ortogonales (es decir, no relacionados) asociados a nuevas vías de desarrollo patológico. En general, han sido producto de la extensión de estudios fisiológicos dirigidos de vías conocidas. En contraste, las nuevas tecnologías están permitiendo la caracterización sistemática, no sesgada, de la variación en los genes, ácido ribonucleico (ARN), proteínas y metabolitos asociados a enfermedades. Los estudios genéticos indudablemente identificarán variables que podrían ser biomarcadores ellas mismas o señalarán marcadores circulantes para una futura exploración. Actualmente es posible examinar cientos de miles de SNPs (Single Nucleotide Polymorphisms o polimorfismos de nucleótido único) en individuos afectados y sanos para buscar asociaciones significativas con la enfermedad. Los estudios de asociación de todo el genoma proveen una revi- sión de variantes en la secuencia genómica, una aproximación que permite revelar nuevas vías patogénicas.

Los datos genéticos también permiten asesorar la causalidad de los biomarcadores en la enfermedad. Si un biomarcador tiene un rol causal, su esperada distribución aleatoria en la población estaría sesgada de acuerdo al estado de la enfermedad.

De las plataformas emergentes para el descubrimiento de biomarcadores, ninguna ha resultado tan interesante como la proteómica y metabolómica. Aún en sus inicios, estas tecnologías ofrecen una mirada complementaria a la complejidad total del fenotipo de la enfermedad. El conjunto de proteínas y metabolitos en una célula puede cambiar rápidamente en respuesta a señales ambientales, de manera que el proteoma y metaboloma (lípidos, azúcares, nucleótidos, aminoácidos y aminas relacionadas, $<2 \mathrm{kDa}$ ) reflejan el estado de una célula o grupo de células en un momento dado. Esta dificultad presenta también un desafío analítico, particularmente aplicada a la búsqueda de biomarcadores en la sangre.

La aplicación de espectrometría de masas y técnicas relacionadas se basa en los esfuerzos por entender y diagnosticar errores congénitos del metabolismo, este trabajo ha culminado en el "screening" neonatal universal que permite la identificación de desórdenes en la oxidación de ácidos grasos, acidemias orgánicas y aminoacidopatías, en muchas de las cuales una intervención puede tener efectos preventivos y/o terapéuticos beneficiosos. Un análisis global metabolómico o proteómico en enfermedades comunes como la HTA, pudiera similarmente aclarar vías patogénicas que pudieran ser moduladas con dieta o drogas.

\section{Genes e hipertensión}

Se conoce de casos de agregación familiar y enfermedades de herencia mendeliana que presentan dentro de sus características la presencia de HTA, esto demuestra la presencia de un componente genético en la génesis y en la tendencia a presentar $\mathrm{HTA}$, que actualmente se sugiere que va de 30 a $50 \%{ }^{3}$, dependiendo de la interacción con factores ambientales.

Los primeros estudios que buscaron genes candidatos se centraron en el sistema reninaangiotensina-aldosterona, siendo aún un área 
importante de estudio ${ }^{4}$, aducina, adrenoreceptores $\beta$, subunidades de proteína $G$, reguladores de la señalización de la proteína $G$, kinasas asociadas a proteínas $\mathrm{G}$ y Rho kinasas.

\section{Sistema renina-angiotensina-aldosterona}

\section{Angiotensinógeno}

Es el precursor de la angiotensina I, que es cortado por renina en un decapéptido, posteriormente procesado a un octapéptido que es angiotensina II, un potente controlador de la presión y el volumen a nivel del sistema cardiovascular.

Jeunemaitre et $\mathrm{al}^{5}$ en 1992 publicaron polimorfismos en el gen del angiotensinógeno, entre ellos T235, que condicionaban mayor riesgo para presentar HTA, en especial al presentarse en homocigosis ${ }^{6}$, y estos polimorfismos difieren dependiendo de la población estudiada. En Chile, Fardella y cols ${ }^{7}$, estudiando 64 pacientes con HTA, compararon los niveles de renina plasmática y aldosterona, y posteriormente buscaron el polimorfismo T235, que produce la codificación para una treonina en vez de metionina en el aminoácido 235 , encontrando una alta frecuencia $(72,7 \%)$ para este alelo, pero no hubo diferencias estadísticamente significativas entre las concentraciones de renina y aldosterona, HTA y la presencia de T235.

Say et $\mathrm{al}^{8}$ estudiando 101 pacientes hipertensos de Malasia, encontraron diferencias estadísticamente significativas en los portadores de este alelo, pero no había diferencias en los niveles de la actividad de la renina plasmática.

Otra variante es T174M, pero diversos estudios no han logrado establecer una diferencia para esta variable .

\section{Renina}

Diversos estudios han buscado diferencias en la actividad de la enzima renina con HTA, obteniendo resultados diversos ${ }^{10}$. Mansego et $\mathrm{al}^{11}$ estudiaron dos poblaciones españolas utilizando 14 SNPs, encontrando diferencias en el SNP rs5707 en las mujeres postmenopáusicas, pero no en otros grupos.

\section{Enzima convertidora de angiotensina}

Su función es hidrolizar angiotensina I en angiotensina II, además inactiva a bradicinina que es un potente vasodilatador.
Este año, Johnson et $\mathrm{al}^{12}$ estudiaron dos SNPs, rs4290 y rs7213516. Estos alelos tienen distinta frecuencia dependiendo de las etnias estudiadas, siendo más alta en afroamericanos (16\%), que en hispanos $(4 \%)$ o caucásicos $(<1 \%)$, estos polimorfismos se asocian a mayor frecuencia de HTA y también a mayor riesgo cardiovascular.

\section{Receptor 1 de angiotensina II}

Bonnardeaux et $\mathrm{al}^{13}$ estudiaron cinco polimorfismos (T573 --> C, A1062 --> G, A1166 --> C, G1517 --> T y A1878 --> G) encontrando una diferencia estadísticamente significativa sólo en A1166-->C comparándolos con normotensos. Este hallazgo ha sido corroborado en otros estudios $^{14}$.

\section{Estudios de secuenciación del ADN}

Se conocen casos con agregación familiar y enfermedades de herencia mendeliana que presentan dentro de sus características hipertensión arterial (HTA), lo que demuestra la presencia de un componente genético en la etiología y en la tendencia de presentar HTA, dependiendo de la interacción con factores ambientales.

Los estudios de secuenciación del ADN, búsqueda de polimorfismos y variantes a través de polimorfismos de nucleótido simple (su sigla en inglés SNPs), se han utilizado para buscar asociaciones con enfermedades multifactoriales o complejas. Para tales fines se han creado coaliciones de grupos de investigación para contar con la mayor cantidad de pacientes y así obtener información más certera sobre las variantes encontradas y la correlación clínica que se busca.

En el año 2005, el consorcio británico de hipertensión y genética (BRIGHT) ${ }^{15}$, buscó asociaciones entre la presencia de HTA y polimorfismos del gen WNK1, responsable del síndrome de Gordon, enfermedad autosómica recesiva, caracterizada por pseudohiperaldosteronismo tipo II e HTA, donde $\mathrm{WNK} 1$ regula la recaptación de $\mathrm{Na}+$ y $\mathrm{Cl}-$ en las porciones distales del nefrón. Se estudiaron pacientes hipertensos sin el síndrome, observando que no existía relación entre polimorfismos para WNK1 y la presencia de HTA, pero sí en la severidad de ésta cuando estaba presente.

Este año, Newhouse et $\mathrm{al}^{16}$, utilizando los nuevos SNPs publicados por el Haplotype Mapping Project, reclutaron información de diversos 
estudios, incluyendo MRC BRIGHT, HYPEST, provenientes de Estonia, LOLIPOP del Reino Unido, el estudio Whitehall I y II de Inglaterra, ELSA de Inglaterra, buscando SNPs que se relacionaran con HTA y excreción de potasio urinario. Utilizaron 17.000 pacientes del estudio BRIGHT y estudiaron 26 SNPs relacionados con WINK1, estando 7 ubicados en el promotor o en el intrón 1, especialmente el SNP rs765250 alelo A (A/A+A/G versus $G / G)$, estando asociados a variaciones de la presión arterial e hipertensión, luego buscaron este SNP en los pacientes de los otros estudios, conservándose la intensidad de la asociación con presión arterial sistólica e hipertensión y una tendencia con la presión arterial diastólica. Posteriormente, se analizó la presencia de SNPs con la concentración de electrolitos en sangre y orina, existiendo una relación entre diversos SNPs y la excreción urinaria de $\mathrm{K}+$, no siendo significativa para otros electrolitos.

En el año 2007, el Framingham Heart Study ${ }^{17}$, que recolecta información en Boston desde el año 1948, seleccionó 2.500 pacientes hipertensos para el estudio genético, encontrando asociación de los genes MEF2C (factor promotor de los miocitos 2C), SYNE1, y TNFSF11, relacionados con el tono arterial. Otro gen candidato es COL8A1, que codifica para colágeno tipo VIII, producido por las células endoteliales en la arteria aorta. Con una asociación menor se encontró a los genes CCL20, CDH13 y LPP. Evaluando tonometría se evidenció una asociación reducida también con los genes LOXL2, SYNE1, y MEF2C, donde LOXL2 codifica para una lixiloxidasa relacionada con la unión de colágenos con elastina, por ende, con la elasticidad arterial, SYNE1 participa en la diferenciación de las células musculares de la aorta y MEF2C en la morfogénesis cardíaca y remodelación extracelular.

En el mismo año, el Wellcome Trust Case Control Consortium (WTCCC) ${ }^{18}$, que reúne información de 50 laboratorios del Reino Unido, publicó sus resultados en la búsqueda de polimorfismos para 7 enfermedades, incluyendo HTA. Este estudio no encontró los polimorfismos previamente descritos en HTA, pero sí con rs2820037 en la región 1q43, los genes más cercanos son: RYR2, cuya mutación produce taquicardia ventricular polimórfica inducida por estrés, CHRM3, un receptor muscarínico y $\mathrm{ZP} 4$, gen para una glicoproteína de la zona pelúcida.

En el año 2009, se publicó el informe del Global Blood Pressure Genetics (Global BPgen) Consortium ${ }^{19}$ que estudió cerca de 35.000 pacientes europeos con ancestros caucásicos, encontrando 8 genes candidatos.

Tabla 2. Resumen de marcadores genéticos identificados en pacientes hipertensos

\begin{tabular}{|llll|}
\hline Polimorfismo & Gen & Ubicación & Mayor riesgo de HTA en: \\
\hline T235 & Angiotensinógeno & $1 q 42-q 43$ & Homocigosis. \\
rs5707 & Renina & $1 q 32$ & Mujeres menopáusicas \\
rs4290 y rs7213516 & Enzima convertidora de angiotensina & $17 q 23.3$ & $4 \%$ población hispana \\
A1166-->C & Receptor 1 de angiotensina II & $1 p 36.22$ & Población general \\
rs765250 & WNK1 & $12 p 13.3$ & Hipertensos severos \\
rs770189 & MEF2C & $5 q 14$ & Población general \\
rs1322512 & SYNE1 & $6 q 25$ & Población general \\
rs10507514 & TNFSF11 & $13 q 14$ & Población general \\
\hline rs792833 & COL8A1 & $3 q 12.3$ & Población general \\
rs2820037 & RYR2 & $1 q 43$ & Población general \\
rs2681472 & ATP2B1 & $12 q 21.3$ & PA sistólica, diastólica e HTA \\
rs1004467 & CYP17A1 & $10 q 24$ & PA sistólica \\
rs7537765 & NPPA; NPPB & $1 p 36$ & Mayor expresión en hígado \\
\hline rs1378942 & CSK & $15 q 24$ & PA diastólica \\
\hline
\end{tabular}


En el gen ATP2B1 se encontraron varios SNPs, tanto para HTA sistólica, diastólica y ambas, siendo el más importante rs2681472.

Para HTA sistólica, el con mayor poder de asociación fue el polimorfismo en 10q24, próximo a CYP17A1, perteneciente a la familia del citocromo P450, que codifica para una enzima participante en la vía de los mineralocorticoides. El segundo polimorfismo se encontró en 1p36, próximo a diversos genes, la mayor señal se encuentra en rs7537765, una variante codificante que se relaciona con mayores concentraciones de homocisteína, preeclampsia e hipertensión variable. En la misma región se encuentran los genes NPPA y NPPB que codifican para el péptido auricular natriurético, ampliamente estudiado como gen candidato en la HTA, MTHFR que codifica para una enzima participante en la síntesis de ácido fólico, AGTRAP que regula negativamente a angiotensina II y la señal más débil fue para CLCN6, que codifica para un canal de cloro. El tercer polimorfismo estaba en la región $17 \mathrm{q} 21$, localizado en el intrón de PLCD3 perteneciente a la familia de fosfolipasa 3 , presente en la túnica muscular de las arterias, participando en la señalización de angiotensina II y endotelina.

Para HTA diastólica, la señal más fuerte fue en el polimorfismo de rs1378942 ubicado en el intrón de CSK (c-src tirosinquinasa) en 15q24. En esta región se encuentran además los genes CYP1A2, pertenecientes a la familia de los citocromos P450, relacionados al metabolismo de la cafeína, LMAN1L codifica para una proteína participante en el transporte intracelular y ARID3B codifica para una proteína que se une a sitios ricos en AT en el ácido desoxirribonucleico (ADN), siendo su mutación letal en períodos embrionarios. El segundo SNP es rs16998073, ubicado en 4q21, próximo a FGFR5, perteneciente a la familia de receptores fibroblásticos, que estimula el crecimiento de diversos tipos celulares, incluyendo células miocárdicas y endoteliales. El tercer SNP es rs653178 en 12q24, localizado en el intrón del gen ATXN2, este SNP está relacionado con otro SNP (rs3184504), que produce un error del marco de lectura en el exón 3 del gen $\mathrm{SH} 2 \mathrm{~B} 3$, que se ha asociado a diabetes tipo 1, enfermedad celiaca, eosinofilia, infarto al miocardio e HTA, este gen se expresa en células hematopoiéticas y endoteliales. El cuarto SNP es rs 1530440 en 10q21, ubicado en C10orf107, un inicio de marco de lectura de fun- ción desconocida, próximo a los genes ARID5B, que es un factor de transcripción en regiones ricas A-T, altamente expresado en tejidos cardiovasculares relacionados a la diferenciación de las células del músculo liso, TMEM26 (proteína de transmembrana 26), RTKN2 (un efector RhoA GTPasa), RHOBTB1 (RhoBTB GTPasa), la familia de las Rho GTPasas, que convierten GTP a GDP y han sido previamente relacionadas con la hipertensión sensible a sal ${ }^{20}$. El quinto SNP es rs16948048 en 17q21, ubicado próximo a los genes ZNF652 y PHB, ninguno de ellos ha sido previamente relacionado con HTA.

\section{Conclusiones y perspectivas}

Gracias al proyecto del genoma humano y los avances en el conocimiento de la fisiopatología en la hipertensión esencial, se han descubierto nuevos biomarcadores. Es poco probable contar en un futuro cercano con un marcador universal, siendo más probable la utilización de una serie de marcadores que otorguen una susceptibilidad específica a una persona, la cual será de vital importancia a la hora de diseñar una terapia personalizada, que incluya un régimen alimenticio, medidas a evitar e incluso la elección de los fármacos qué serán más efectivos.

\section{Referencias}

1. Ministerio de Salud. Encuesta Nacional de Salud Chile, 2003.

2. Cruz-Coke R, Donoso H, Barrera R. Genetic ecology of hypertension. Clin Sci Mol Med 1973; 45: 55s-65s.

3. Marteau JB, Zaiou M, Siest G, Visvikis-Siest S, Genetic determinants of blood pressure regulation. J Hypertens 2005; 23: 2127-43.

4. Martínez-Aguayo A, Fardella C, Genetics of Hypertensive Sindrome. Horm Res 2009; 71: 253-9.

5. Jeunemaitre X, Soubrier F, Kotelevtsev YV, Lifton RP, Williams CS, Charru A, et al. Molecular basis of human hypertension: role of angiotensinogen. Cell 1992; 71: 169-80.

6. Giménez-Roqueplo AP, Jeunemaitre X. Genetics and essential hypertension: candidate genes or screening of the whole genome?] Arch Mal Coeur Vaiss 2003; 96: 1089-95.

7. Fardella CE, Claverie X, Vignolo P, Montero J, Villarroel 
L. T235 variant of the angiotensinogen gene and blood pressure in the Chilean population. J Hypertens 1998; 16: 829-33.

8. Say YH, Ling KH, Duraisamy G, Isaac S, Rosli R. Angiotensinogen M235T gene variants and its association with essential hypertension and plasma renin activity in Malaysian subjects: a case control study. BMC Cardiovasc Disord 2005; 5: 7.

9. Fernández-Llama P, Poch E, Oriola J, Botey A, De La Sierra A, Revert L, et al. Genetic polymorphisms of the renin-angiotensin system and essential hypertension. Med Clin (Barc) 1999; 112: 561-4.

10. Jeunemaitre X. Genetics of the human renin angiotensin system. J Mol Med 2008; 86: 637-41.

11. Mansego ML, Redon J, Marín R, González-Albert V, Martin-Escudero JC, Fabia MJ, et al. Renin polymorphisms and haplotypes are associated with blood pressure levels and hypertension risk in postmenopausal women. J Hypertens 2008; 26: 230-7.

12. Johnson AD, Gong Y, Wang D, Langaee TY, Shin J, Cooper-Dehoff RM, et al. Promoter polymorphisms in ACE (angiotensin I-converting enzyme) associated with clinical outcomes in hypertension. Clin Pharmacol Ther 2009; 85: 36-44.

13. Bonnardeaux A, Davies E, Jeunemaitre X, Féry I, Charru A, Clauser E, et al. Angiotensin II type 1 receptor gene polymorphisms in human essential hypertension. Hypertension 1994; 24: 63-9.

14. Kainulainen K, Perola M, Terwilliger J, Kaprio J, Koskenvuo M, Syvänen E, et al. Evidence for involvement of the type 1 angiotensin II receptor locus in essential hypertension. Hypertension 1999; 33: 844-9.

15. Newhouse S, Wallace C, Dobson R, Mein C, Pembroke J, Farrall M, et al. Haplotypes of the WNK1 gene associate with blood pressure variation in a severely hypertensive population from the British Genetics of Hypertension study. Human Molecular Genetics 2005; 14: 13.

16. Newhouse S, Farrall M, Wallace C, Hoti M, Burke B, Howard P, et al. Polymorphisms in the WNK1 Gene Are Associated with Blood Pressure Variation and Urinary Potassium Excretion. PLoS ONE; 4 (4): e5003. Epub 2009 Apr 4.

17. Levy D, Larson M, Benjamin E, Newton-Cheh C, Wang T, Hwang S, et al. Framingham Heart Study $100 \mathrm{~K}$ Project: genome-wide associations for blood pressure and arterial stiffness. BMC Medical Genetics 2007; 8 (Suppl 1): S3.

18. The Wellcome Trust Case Control Consortium. Genome-wide association study of 14,000 cases of seven common diseases and 3,000 shared controls. Nature 2007; 447 (7145): 661-78.

19. Newton-Cheh C, Johnson T, Gateva V, Tobin MD, Bochud M, Coin L, et al. Genome-wide association study identifies eight loci associated with blood pressure. Nat Genet 2009 May 10.

20. Du YH, Guan YY, Alp NJ, Channon KM, Chen AF, Endothelium-specific GTP cyclohydrolase I overexpression attenuates blood pressure progression in saltsensitive low-renin hypertension. Circulation 2008; 117: 1045-54. 